\title{
Within Bounds and Between Domains: Reflecting on Making Tea within the Context of Design Elicitation Methods
}

\author{
mc schraefel and Alan Dix ${ }^{2}$ \\ ${ }^{1}$ IAM Group, ECS, \\ Umiversity of Southampton \\ \{mcttea\}, http://www.ecs.soton.ac.uk/ mc \\ ${ }^{2}$ Computing Department, \\ Lancaster University \\ \{alan@hcibook.com\}, http://www.hcibook.com
}

\begin{abstract}
Making Tea is a design elitation method developed specifically to deal with situations in which (1) the designers do not share domain or artefact knowledge with design-domain experts, (2) the processes in the space are semistructured and (3) the processes to be modeled can last for periods exceeding the availability of most ethnographers. We propose a set of criteria in order to understand why Making Tea worked. Through this criteria we also reflect upon the relation of Making Tea to other design elicitation methods in order to propose a kind of method framework from which other designers may be assisted in choosing elicitation methods and in developing new methods.
\end{abstract}

\section{Introduction}

Making Tea was a method used to elicit processes and knowledge form domain experts in the design electronic lab book for Chemists. This domain has proved difficult in previous reported work, yet the use of an acted out tea making analogy led to a successful design [19].In this paper we wish to reflect on the design method Making Tea in order to reflect upon its efficacy as a design elicitation method. In other words, we wish to look at why Making Tea works for design elicitation and validation. In carrying out this exercise in reflection, we contextualize Making Tea in terms of four categories: neutral territory, boundary representations, disruption, and time compression. While this abstraction of Making Tea from practice to model has allowed us to address the questions "why did this method work? Can it generalize?" the categorization has also let us reflect upon the properties of other design elicitation methods against these categories. Such an analysis or start at a possible taxonomy of methods, we hope, may have two outcomes: first, to help designers choose techniques to support their design constraints and second, to help the development of new design methods for evolving off-the-desktop environments. In the following sections, therefore, we briefly overview the Making Tea method, from which our assessment categories emerge. We then present a discussion both of Making Tea and related design elicitation methods in terms of these categories. We then look at a recent application 
of Making Tea to a new domain to show how the attributes of the method we've described can be generalized beyond the founding domain for the method. We conclude with thoughts on evolving new methods. Related work is discussed in context throughout.

\section{Making Tea}

Making Tea [19,20] evolved as a design elicitation and validation method when a group of computer scientists were faced with the challenge of having to design a digital version of a synthetic chemist's lab book. The new artefact was requested as part of a movement in EScience to get more of the experimental record to be generally available within a lab locally and within the community globally. Heretofore, the paper-based lab book had restricted access to the data to whomever had the single copy of the book itself. The fear of engaging in this process was twofold: first, there were numerous previous efforts to develop digital lab books, the success rate of which in terms of take up had been small; second, the computer scientists taking on this task had very little chemistry knowledge (beyond highschool/A levels). Without this domain expertise, it became readily apparent that while the team could observe the lab environment and the types of activities taking place (no room for lab books; many people working at once; one chemist running multiple experiments) as well as gain from interviews knowledge of the types of roles the lab book played (individual record, legal document for intellectual property, communal archive), getting at the critical issue - when, how and why certain things were recorded and others were not was out of reach. The reason for this inaccessibility was simple: chemistry is a highly expert, abstract process. It requires deep domain knowledge in order to design an experiment, understand the chemical processes involved, and those the chemist desired to emerge. Without this understanding however, task analysis for instance, of the experimental recording process could not take place, and without such a model against which to design, the likelihood of developing a successful artefact went close to nil.

In frustration, among the team of Canadian and British computer scientists and chemists, the group made tea, a process embraced by both nations for restoring the soul. It was at this point that the chemist-turned-software-engineer on the team said "Making tea is much like doing an experiment." The rest is history. The design team took up the observation and used making tea to model the process of both carrying out an experiment and recording it. To wit, the team's chemist make tea multiple times: first using well understood kitchen implements, where questions were asked like "why did you not record that?" "You just did 20 steps to get the tea ready to pour, yet you've only written down "reflux." Why?" From kitchen implements, the team moved to chemistry apparatus set up in the team's design space. From there the team moved to the chemistry lab. The results of the exchanges in these spaces informed the design process. Indeed, they also informed the validation process: design reviews with chemists in various positions, from researchers to managers to supervisors, were carried out by making tea, and demonstrating how the artefact worked in the tea-making 
experimental process. This time it was the chemists' turn to interrupt the presentation with questions about the artefact and their process.

Finally, the making tea model of the process was used to support the design of the middleware for the project. Once this model was worked out, it was then used successfully to test other actual chemistry experiments to see whether it generalized to more intrigued experimental cases. From the Making Tea approach, we developed both front end and middle ware solutions for the lab book problem that tested well.

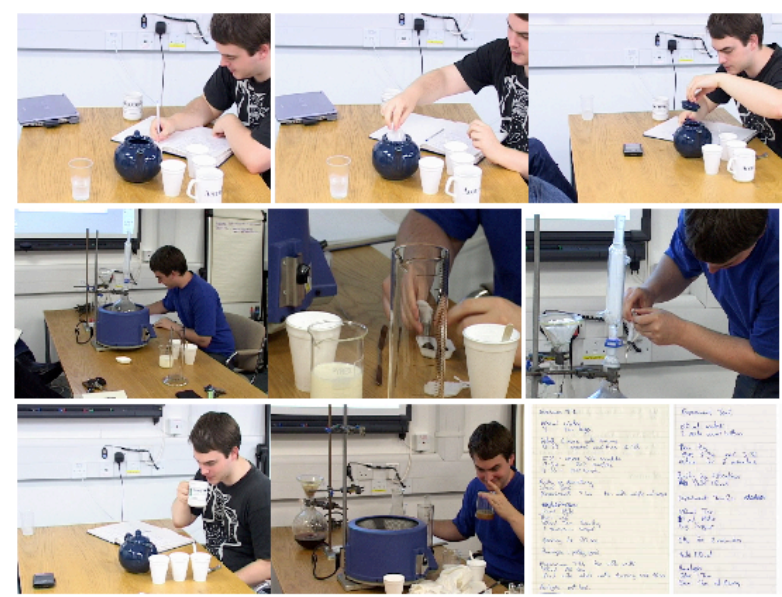

Fig. 1. Team chemist making tea as an experiment with domestic equipment, top row then with lab apparatus, second row. Bottom row, left and middle, evaluating the state of the completion of one process. The paper lab book entries are lower right.

\subsection{Attributes of Well-Made Tea}

From the above description, it is easy to hear resonances with other design elicitation methods, from participatory design to artefact walkthroughs. Comparison with these and other elicitation methods have been detailed previously [20]. Suffice it to say for our purposes here that the core distinguishing feature between Making Tea and a variety of elicitation methods is that other methods, from scenarios to story telling to cultural probes implicitly assume that there is either already a shared understanding of the artifacts/processes to be modeled by a designer, or that that knowledge can be gained (as in Mockups and Apprenticeships) readily through direct practice. Likewise, other design methods tend to assume, implicitly, that the process for design can be observed in its entirety in a reasonable period. These implicit assumptions are appropriate for the conditions under which they have been applied. In our case, however, we were in a domain where expertise or domain knowledge was neither shared between team and experts, nor could it be grasped without possibly a considerable period of study. Similarly, actual chemistry experiments can take months to years to complete. 
Making tea presented a model of a process that was readily understandable and interogatable by the design team in particular during elicitation and by the chemists in particular during design reviews. Making Tea also gave us a representation of a complete process within a brief enough period of time that the process could be readily repeated, and at increasing levels of fidelity. In the chemistry wet lab context in particular, the Making Tea analogue of the experiment allowed us to carry out a process in a safe environment. The analogue itself helped us elicit with the experts both the similarities of actual experiments to tea as well as teasing out critical differences (spill tea, wipe it up and wring out the cloth; spill compound, get a scraper and get it off the table, wood chip and all, and process it till that precious bit of compound is retrieved).

In considering why Making Tea worked as effectively as it did, we propose four attributes for reflection: (1) the territory of the design space; (2) the use of boundary representations; (3) disruption of practice and (4) time compression. These aspects are not unique to Making Tea, but the need to develop a method to address the gaps for this kind of domain which tea addresses (highly expert, longitudinal, semistructured, without shared domain knowledge) helps make explicit the practices of design elicitation methods in general. In the next section we consider how these attributes are reflected both in Making Tea and in more well known methods.

\section{Making Sense of Making Tea}

In order to consider why Making Tea worked as a design elicitation/validation method, we consider the following five criteria:

- Design territory - Making Tea created what we call "neutral territory" a space that was not 'owned' by either designer or users

- Boundary representation - Building from this, Making Tea by being understood by both designers and users, formed a point of contact.

- Disruption - By being similar yet different from the actual process being represented, Making Tea forced the users to reflect on their tacit activities.

- Time compression - Making Tea reduced the time taken for a normal experiment into a period that could be completed in a participatory session.

We will first see how these contributed to Making Tea and then look at each again in the context of the broader design process and related techniques.

Neutral territory. The most traditional participatory design aspect of Making Tea is the development of the founding analogy. Design team members brainstorm with design experts to develop an analog with which both communities feel comfortable, and which the domain expert community in particular feels is a credible analog for the process to be modeled. In the case of Making Tea with chemists, the analogy was developed between team and resident domain expert then proposed to experts in the chemistry community at large. So there was a high degree of confidence in the credibility of the chosen analog. It was also discovered that the analog had long standing resonances in the community, which added another attribute of both the familiar and 
the legitimate ${ }^{1}$. By leveraging an ongoing debate in the community (which goes first: the milk or the tea) this prompted a willingness on the part of those not involved directly in the project to tell their own stories about why and how making tea reflects chemistry. The process of developing the analogy creates a sense of co-ownership from the start of the process.

Added to this, the familiarity of tea, its very commonness, created a kind of ironic humour for the design team and domain expert. "We are really talking about something real and important, and we're doing it by making tea. That's bizarre." The resulting playfulness of the analogy created a relaxed atmosphere during the sessions and increased confidence for the designers as beginning to understand the process. Likewise, the domain experts had fun translating "proper" chemistry into a discussion about tea. From this relaxed conversational space, discussion about the lab book, the artifact under consideration, as much as the experimental process, took place in a focused but relaxed set of exchanges.

For the chemist, the analogy of making tea as an experiment immediately resonated with the ongoing debate in the community of which to add first: the tea or the milk.

Boundary representations. One problem with the chemistry lab. is the expertise needed to understand its operation. While it may be possible to have a domain expert run the analogous process for interrogation by the design team, without the design team having first-hand experience of the real domain environment, it would be a far less rich experience. In the Making Tea experience, when the analogy was first developed, half the team had been to the chemists' lab and half had not. The difference to the understanding of the analogy on the rest of the team's part once contextualized by their spending time at the real lab was reflected upon as "night and day."

This is not to say that Making Tea was entirely separated form the real laboratory. Indeed, pictures from the real environment were regularly before the designers at the project web site. Both the memory of the real lab and these photographic reminders informed the creation of the neutral territory of the tea-making space. The crucial thing is that it produced a setting in which both the designers and chemists were able to bring their differing understandings and hence share and learn.

Making Tea is fundamentally an interaction design elicitation technique, but it was also used to model the process that would be emulated by the pervasive system being designed to support it. Because the analogy was sufficiently faithful, the software engineers on the design team could translate the process first into an abstract model and then, in current work, into an architecture to support the described process. [19]. That is it not only offered a way for domain experts to describe their tasks and activities, but also one where software engineers could offer back potential new designs.

Disruption. As noted the very commonness of Making Tea also contributed to a sort of playful strangeness. The serious and careful process of laboratory work was suddenly translated into the fun, carefree and social exercise of tea brewing.

\footnotetext{
${ }^{1}$ See the Smart Tea site, http://www.smarttea.org, for a selection of articles by chemists arguing about how to make tea, including one by George Orwell. The project kicked off on the anniversary of this broadside to chemists on tea making.
} 
The context of tea provided opportunities to go off on tangents about the lab book that would have been difficult to get at by interviews alone. This atmosphere carried forward from the design elicitation sessions with the domain expert running the tea experiments into the design review sessions with the participant chemists. Tragicomic stories of how lab book pages have been nearly lost and then found again, for instance, came pouring forth in the relaxed atmosphere of both elicitation and review sessions: it felt safe to talk about such matters. While funny, the anecdotes also helped reveal artefact problems that our design would need to address. The depth and breadth of these conversations was particularly valuable considering the expertise gap. Comments from the chemists about the analogy, such as "I would never have thought about that [process attribute] if I hadn't had to explain why it was different from this [making tea]. That's interesting" were gold for the design process.

For the non-chemist, imagining having to write down every step in making a cup of tea while making the tea communicated the need for assistive technology, the understanding why a process might be under-documented (as it seemed to be), and the problem of physically placing the artifact in the lab/kitchen.

Time compression. In the real laboratory, experiments take anything from hours to weeks to complete. In contrast good tea brewing is over in a few minutes. The time compression itself afforded by the technique let us iterate rapidly on both observations of process and versions of the design. Iterations while always sought are something but can be prohibitively costly. In Making Tea, the iteration was cheap and highly valuable. Indeed, the richness of the information developed from the combined field studies and tea making experiment yielded rich, functional/task analyses that were modelled into early prototypes. Getting the functional attributes modelled early left more time to focus on the analysis of some of the potentially more ephemeral, experiential attributes of the artifact-in-use. In the following sections, we look at each of these attributes of Making Tea in the context of related design methods.

\subsection{Neutral territory}

The Designer as Interloper. Design involves the meeting of two worlds: the domain world of the user replete with processes, artefacts, aspirations, and nuanced interactions, and the world of the designer with technical and/or aesthetic knowledge, techniques, tools experience of other design meetings (Figure 3 ).

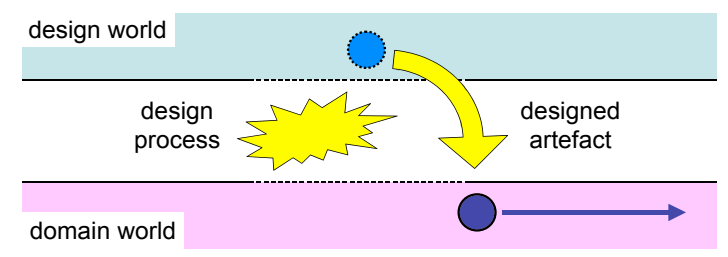

Fig. 3. Interaction between design and domain worlds

But this meeting of design world and domain world is not symmetric. The designer will leave, like a silent stranger in a spaghetti western walking into the sunset. And 
like the spaghetti western hero (or anti-hero) the designer disrupts, exposes hidden problems, sorts out difficulties. The designer may revisit for enhancements or maintenance, but often is gone forever. Of course, the purpose of design is precisely this leaving; it is the thing that is left behind, the intervention, whether technical artefact, revised process, or socio-technical system, that is the desired end point.

Moving between Worlds. Design methods differ in how they manage this necessary meeting between the design and domain worlds. In traditional HCI design methods such as task analysis [4] and in software engineering methods such as UML the aim is to capture the domain world in a formal or informal representation, bringing the 'relevant' aspects of the domain world into the design world. To do this the designer 'visits' the domain: interviews users, reads documentation, perhaps even observes using ethnographic or other field study techniques (Figure 4). The representation may be a formal hierarchical task analysis [1] or cognitive model [6, ch.12]. or a more informal rich picture as in the Soft Systems Method [2], scenarios or personae.

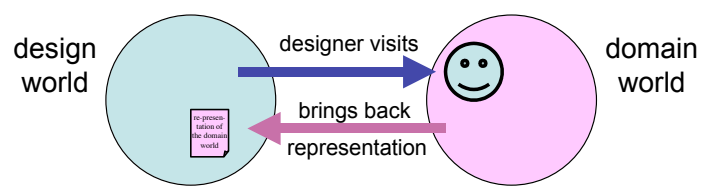

Fig. 4. Movement between worlds in user-centered design.

In contrast various methods including traditional brainstorming and participatory design methods such as PICTIVE [15] seek to invite the future users into the design world. Whereas the role of the user-centred designer is to suck out the pertinent aspects of the domain from the users and re-present this in the design space, the role of the participatory designer is instead to share out the critical aspects of the design space, to present the available options, the pertinent technology, and the fruits of previous design experience in a way that is comprehensible to the user community.

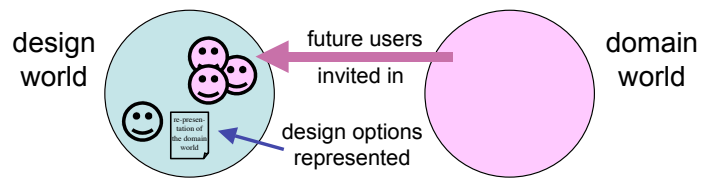

Fig. 5. Movement between worlds in participatory design.

Finding common ground. Making Tea falls somewhere between the above methods. By using analogy for both expert elicitation and design testing, Making Tea is neither a full moving into the wilds of the domain world, nor a bringing of the stakeholders fully into the design world. Instead making tea creates a middle ground, a third world that forms a neutral space for design discussion among designers and stakeholders (Figure 6). It is far enough away from the expertise of the domain world to be comprehensible to the designers and to force reflection by the users, but still clearly a domain-oriented position 


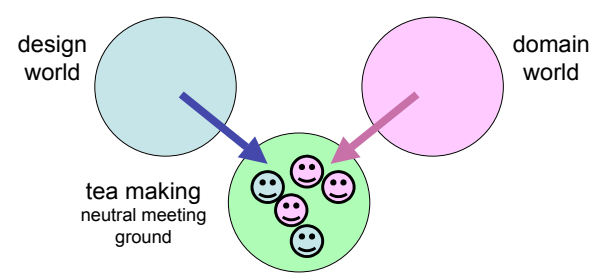

Fig. 6. Movement into neutral territory: Making Tea

Looking at this claim for Making Tea's neutrality we can see that there is a continuum here (Figure 7). The chemists were asked to make tea using kitchen equipment outside their laboratory. The 'chemistry' setting was very much one of the users, but the physical setting and implements were deep into the design world. In contrast, the act of making tea with laboratory equipment was closer to the domain world. One could imagine asking the chemists to make tea in their own laboratory with their own equipment. This would almost certainly have broken every health and safety guideline, but would have stepped even closer towards the chemists territory, yet still have had some of the elements of neutrality.

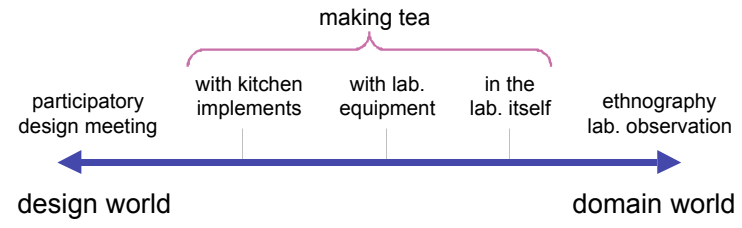

Fig. 7. Neutrality continuum

While we have placed participatory design at one end of this spectrum, Muller argues that participatory design is itself a 'Third Space' between system designers and end users [16]. Certainly some PD practices would fit in the middle ground of this continuum, for example, the use of drama and story. The use of a metaphorical vehicle, in our case of Making Tea is particularly important where there is little common ground because of esoteric or complex domain knowledge. However, in domains, such as office work, there is often enough mutual understanding for more traditional methods to bridge the gap. Of course, as well as the neutrality in terms of knowledge and expertise, the tea making also encouraged a neutrality of ownership which is also common to participatory design.

\subsection{Boundary Representations}

We have seen how the communicative nature of neutral space forms a point of contact between designers and users, especially important when, as in this case, there is little overlap of knowledge and the domain is specialised. In studies of work domains ethnographers frequently notice boundary objects, artefacts and representations that mediate between individuals and groups. The act of making tea is such a boundary representation, making the process of the chemistry laboratory available to the user and allowing design concepts to be communicated to users. 
Indeed the meetings between worlds in the design process is not so far from those between amateurs and professionals in Star and Griesemer's original study of boundary objects [21]; the various forms of representation, diagrams and notations used to facilitate communication between designers and users are perhaps natural parallels of taxonomies and categories used as points of 'shared' (but also disparate) meaning within a museum. Just as in the museum context we have a meeting of disparate communities of practice (designers, users, perhaps clients, implementers) who together form a community of interest (getting a system running and used). This analogy has also been noted in the Agile Development and Information Architecture communities where boundary objects are being actively discussed in conferences and web logs (e.g. [14]).

Of course any design method that has any claim to involve users will use some forms of boundary representation. In very traditional design methods formal representations may be used both within the design team, but also to communicate with users. For example, hierarchical task analyses and business process diagrams are both used as both analytic and communicative notations. Even traditional computer flow diagrams have been to discuss the interface dialogue with users and state transition diagrams can even be found in the user documentation of digital watches [6, ch.16].

Looking at richer and less formalised methods we can see other boundary representations. Scenario-based methods are of course powerful for envisioning design options within the design team, but also the scenarios can be used to discuss them with users. Personae too can be used as 'surrogate' users during design and also can be discussed with users themselves.

Various forms of storytelling, narrative and drama have been used in design process both for elicitation and also communicating the results of design (e.g. [11]). In a similar vein the UTOPIA project used professional actors and production to create a collection of short films relating experience of older people using IT [5]. This was designed in this case to create a means of communicating the needs of older users to (often predominantly much younger) software designers.

Whilst people skilled at constructing narratives can be brought in to help develop the story so that all stakeholders in the design process are on the 'same page, in Mak-

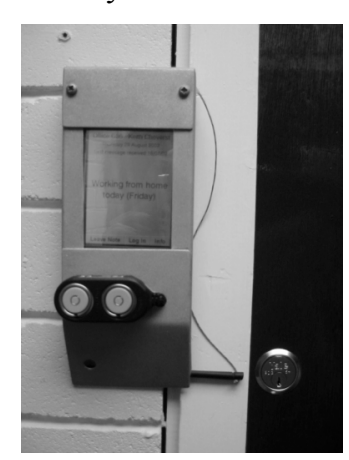
ing Tea, the vividness of the analogy itself helped to 'tell the story' of the lab book, if in a compressed fashion

As designs are developed storyboards, video envisionments, prototypes and even virtual reality simulations are used to communicate design ideas with users. An extreme form of this is when putative systems are deployed in medium to long term in actual use environments. These may be advanced prototypes intended as early roll-out, but may also be used as part of elicitation.

Fig. 8. Hermes door display [3].

The Hermes project is one example of this. Small displays have been placed outside office doors where electronic notes can be left by the occupant and visitors, and accessed by the occupant via web, email and SMS gateways (Figure 8). The long term installation of a number of these has led to insights that a short term experiment, storyboard or scenario would have missed [3]. 
A similar example is the FXPAL's investigation of Personal-Interaction Points (PIPs). Small RIFID cards identifying individuals were used to enable printers and smart meeting rooms to personalise their interfaces for the user and offer sensible default options (e.g. print document or show presentation that you have recently been working on). This was deployed over a substantial period again allowing an understanding of which features were really useful in practice [22].

Interestingly both Hermes and the PIPs were deployed in the designers' own work environment - amongst users who understood the project and where the designers were amongst the users. This clearly need not be the case but experimental deployments clearly need 'friendly' user groups.

Boundary representations during elicitation vary in their ecological felicity - the extent to which the representation is a realistic reflection of practice. In the case of Making Tea the neutrality continuum effectively corresponds to levels of ecological validity. Similarly an ethnographer's account of observed practice would be more ecologically valid than a made-up scenario. Note that we are not regarding ecological validity as a value judgement. The kitchen-implement-based tea making was equally useful and produced different insights than the laboratory equipment exercise.

Boundary representations also vary as to their technical felicity, that is the extent to which the representation captures the details of how proposed intervention / artefacts / designs reflect what would actually be delivered. So a storyboard would have less technical felicity than a prototype.

To some extent ecological felicity represents an agreement with the domain world whereas technical felicity represents an agreement with the design world. This may suggest a trade-off between the two and arguably deployed systems such as Hermes have both high ecological felicity and high technical felicity. It is also important to note that neither of these is an absolute scale and a particular boundary representation may be felicitous on some ecological or technical aspects and not in others.

\subsection{Disruption}

One of the ways Making Tea worked is that by being similar but different from normal laboratory work it forced reflection. Because it disrupts normal activity this activity is called into question and becomes available for reflection. This creative use of disruption is something that can be seen in other elicitation and design methods.

Cultural probes are a clear example of this and act as a form of asynchronous elicitation for the designer. The probes are sent out as packs to participants, with postage pre-paid services to return the probes back to their design home. These packs include a variety of provocative items designed to invite users to reflect on their environment and reflect this back to the designers. For example, cultural probe packs used for domestic settings have included disposable cameras to photograph significant things in the home, a plastic beaker to listen at walls and hear what is often ignored, and postcards to write notes [8].

Note how cultural probes invite users to produce boundary representations. If users were simply asked to write about their daily activities then there would be a representation created, but cultural probes do more. The subversive and provocative nature of the materials supplied force different ways of thinking. 
This follows a long tradition in ethnography. Whilst some ethnographies are deliberately non-intrusive, observing ordinary activity, others, like the use of probes, are more intrusive. The aim is to obtain a unique perspective, the subjects own understanding of what they do if they were to reflect on it. Of course for tacit activity, by definition we do not normally reflect on them. The trained external observer can produce such a reflection, but also disruption can be used. In the late 1950s and 1960s Garfinkel conducted a series of "breaching experiments" that were designed, in his own words, to make "commonplace scenes visible". For example, students were asked in ordinary conversation with friends to keep asking for clarification, or when playing board games with subjects experimenters would deliberately ignore rules $[7,12]$.

As thought experiments designers often invent "impossible" objects or ones that defy expectations. For example, Sarah Pennington's design for a mobile phone that has no 'call' or 'receive' buttons [9] and in another RCA design disruptive office furniture is proposed to cause deliberate conflict [13].

Given suitable guidance or props, such as cultural probes or making tea, users can similarly produce innovative views on their own experience. For example, in the PRESPS method users are asked to produce photo-essays, annotated photographic accounts, rather like video diaries, of interesting events, thoughts or things in their own environment [10]. For example one participant described how she flicks her phone open and shut for the satisfying click it makes and how all her friends do the same.

When thinking along such lines it is easy to find other disruptive means to cause reflection, for example, taping shut cupboards or doors in someone's home, or forcing participants to perform domestic activity with mittens on. The nature of laboratory work means that disruption of this kind would be dangerous and hence the safe 'sand box' of tea making was ideal.

This technique of disruption is also used in other disciplines, in genetics with the 'knockout' method or RNAi (RNA interference) genes are removed from DNA in order to understand their effect and in neurology studies of patients who have suffered major trauma (e.g. nails embedded in the brain) have been invaluable in understanding how 'normal' brain activity works.

As a general rule seeing an unusual, broken or close but different version of something allows a fresh understanding of 'normality'. Likewise, disruption may inspire new approaches to achieve that state.

\subsection{Time compression}

Long-term or intermittent activities can be particularly difficult to study. Ethnographies or other observational methods struggle with this kind of process, especially if the periods of activity are short as important aspects of the work may never occur during observed periods. In such cases it is usually necessary to look at written procedures and documentation or reply on interviews or anecdotal elicitation techniques. The former are known to be problematic as the work as documented is often at variance with the work as performed. The latter is problematic because it is often hard to elicit the tacit aspects of an activity.Dramatisation and story telling can again help. 
Story time need not be the same as real time yet the story has a degree of realism provoking reflection on process. The analogous situation in Making Tea adds an extra dimension to this in that the metaphorical task can actually be performed so that it does take place in a foreshortened 'real' time and in some ways because of its real performance can serve to demonstrate tacit skills and knowledge even if the users do not explicitly realise it.

A different method of eliciting these long-term processes has been the studying of artifacts based on the fact that they often encode procedural state and temporal event triggers in their physical disposition in the environment (location, orientation) as well as more explicit marks and annotations. This computational role of artifacts mean that they can be used to decode and deconstruct work processes and as an aid to elicitation with users [18].

\section{Making Tea in Bioinformatics: the Jigsaw Analogue}

More recently, a project has been underway to take the lessons learned from designing digital information capture systems for synthetic chemistry to bioinformatics ${ }^{2}$. The desire was also to see if Making Tea (a) could be applied to a new domain and (b) would be a useful approach to apply in this new space. Making Tea evolved to handle longitudinal, semi structured, highly expert processes. It was after a three hour tutorial in phylogyny that analogy would be useful: while the team gained an understanding of the importance and interestingness of genomics and a new respect for protein, the team could in no way replicate the month to year long processes that, were run it seemed as individually as the scientists doing the work themselves.

The analogy proposed by the bioinformatician on the team has been jigsaw puzzle solving. The analogy was inspired by the work on shared jigsaw puzzle solving strategies developed by Johnson and Hyde [17]. The effort of a bioinformatitan's quest for various genes - isolating them, understanding how they work with other genes and so on - is very much akin to putting a puzzle together -- a puzzle where there is lots of blue sky, some of the pieces do not belong, and edges are few and far between. From time to time, new pieces become potentially available for the puzzle, but these must first be found (sometimes in the same place one just looked the day before) and then analyzed to see if they do indeed fit. Somethat look like they do can be thrown out the next, pending new analysis or pieces. As puzzle solving goes, bioinformatics is extremely existential. The analogy, however, has helped the design team to understand and question not only the practice (when are certain solving strategies invoked; how are these traced; when does one want the traces back through past guesses at part of the solution that have either been kept, discarded or are on hold) but also the combined digital and physical recording practices that are maintained (do you keep your partial solutions in organized spaces, or strewn across related parts? Do you strew because that works over time, or there are impediments to better organization). Questions can be rapidly associated with the puzzle analogy --"what part of the puzzle (process) are we talking about?" - within a time-compressed, observable way.

\footnotetext{
${ }^{2}$ See the myTea project, http://www.mytea.ecs.soton.ac.uk
} 
Here the analogy has again been field tested with domain experts, and again has met with recognition, which has in turn drawn out extensions to the analogy. Experts express new ways in which their processes are like the analogy or are dissimilar from it. Here, the jigsaw analogue has additional strengths: the research on shared jigsaw solving strategies was designed to look at collaborative problem solving strategies. Unlike synthetic chemists who work largely on their own, bioinformatitians collaborate frequently. The research itself on collaborative jigsaw solving also gives us potential insight into the kinds of strategies used in the bioinformatics community: where are they similar? Different? How can they be better supported in the recording process in particular? This of course is a large part of the purpose of the jigsaw solving research: to transfer to other domains. We are simply getting double and concurrent leverage from both the analogy and the results of actual jigsaw solving research.

In sum, in another domain where a design team has been working in the space of semi-structured, high domain expertise, longitudinal practice, use of the Making Tea approach has provided a space in which designers and experts can elicit practice and validate design approaches.

\section{Conclusion}

We have analyzed the proven success of the Making Tea method in order to draw broader lessons for design. We have discussed four interlinked attributes: the importance of neutral space for designers and domain experts to meet, boundary representations for them to share and build knowledge and mutual understanding, disruption forcing domain experts to reflect on their tacit practices and time compression allowing long-term activities to be studied within reasonable observation and design cycles. Each was critical for the success of Making Tea and we have seen how elements can be seen in other methods. Finally we saw how some of these general issues have been reinstantiated in the Jigsaw metaphor. We hope that this work will contribute to the collection of a 'kit bag' of techniques and criteria that can be used by the methodological DIY-er in creating customised methods for problematic settings.

\section{Acknowledgements}

Anonymized

\section{References}

1. Annet, J. Chapter 3: Hierarchical Task Analysis. In The Handbook of Task Analysis for Human-Computer Interaction. Diaper, D. and Stanton, N. (eds.). Lawrence Erlbaum 2004

2. Checkland, P. Systems Thinking, Systems Practice. John Wiley, Chichester, 1981.

3. Cheverst, K., Fitton, D. and Dix, A. Exploring the Evolution of Office Door Displays, In Public and Situated Displays: Social and Interactional aspects of shared display technologies. O'Hara, K. et al. (eds). Kluwer, 2003. pp. 141-169. 
4. Diaper, D. and Stanton, N. (eds). The Handbook of Task Analysis for Human-Computer Interaction. Lawrence Erlbaum Associates, Hillside, NJ, 2003.

5. Dickinson, A., Eisma, R., Syme, A. and Gregor, P. UTOPIA: Usable Technology for Older People: Inclusive and Appropriate. In A New Research Agenda for Older Adults BCS HCI 2002, London 2002 (ed. S. Brewster and M. Zajicek) pp.38-39

6. Dix, A, Finlay, J., Abowd, G. and Beale, R. Human-Computer Interaction, third edition. Prentice Hall, 2004.

7. Garfinkel, H. Chapter 2: Studies of the routine grounds of everyday activity. In Studies in Ethnomethodology. Polity, 1967.

8. Gaver, B., Dunne, T. and Pacenti, E. Design: Cultural probes. Interactions. 6(1), 1999. pp. 21-29.

9. Gaver, W. , Beaver, J. and Benford, S. Ambiguity as a resource for design. Proc. of CHI'2003. ACM Press, 2003. pp. 233-240.

10. Go, K., Takamoto, Y., Carroll, J., Imamiya, A. and Masuda, H. Envisioning systems using a photo-essay technique and a scenario-based inquiry, In Proc. of HCI International 2003. Lawrence Erlbaum Associates, 2003. pp. 375-379

11. Gruen, D. Stories and storytelling in the design of interactive systems. Proc. of Designing interactive systems, New York City, New York, 2000, 446--447.

12. Heritage, J. Chapter 4: The morality of cognition. In Garfinkel and Ethnomethodology. Polity, 1984.

13. Law, A. and Boucher, A. The Disruptive Office Mechanised Furniture to Promote Useful Conflicts. In Proceedings of 1AD: First Intnl Conf. on Appliance Design. 2003.

14. Marick, B., Boundary Objects : An analytical concept from science studies: does it apply to acceptance tests? In Analogy Fest held at the Agile Development Conference, June 25-28, 2003. http://www.visibleworkings.com/analogyfest/

15. Muller, M. PICTIVE - an exploration in participatory design. In Proc. of CHI'99. ACM Press, 1991. pp. 225-231.

16. Muller, M. Participatory design: the third space in HCI. Chap. 54 in J. Jacko and A. Sears (eds.) The Human-Computer Interaction Handbook: Fundamentals, Evolving Technologies and Emerging Applications. Lawrence Erlbaum.2002, pp. 1051-1068

17. Johnson, H., and Hyde, J. Towards modeling individual and collaborative construction of jigsaws using task knowledge structures. In ACM TOCHI, 10.4(2003):339-387.

18. Ramduny-Ellis, D., Dix, A., Rayson, P., Onditi, V., Sommerville, I. and Ransom, J. Artefacts as designed, Artefacts as used: resources for uncovering activity dynamics. Cognition, Technology and Work (to be published 2005)

19. schraefel, m. c., Hughes, G., Mills, H., Smith, G., Payne, T. and Frey, J. (2004) Breaking the Book: Translating the Chemistry Lab Book into a Pervasive Computing Lab Environment. In Proc. of CHI 2004, pp 25-32.

20. schraefel, m. c., Hughes, G., Mills, H., Smith, G. and Frey, J. Making Tea: Iterative Design through Analogy. In Proc. of Designing Interactive Systems, 2004, pp. 49-58.

21. Star, SL. and Griesemer, J. Institutional Ecology, 'Translations,' and Boundary Objects:Amateurs and Professionals in Berkeley's Museum of Vertebrate Zoology, 19071939," Social Studies of Science, 1989, 19:387-420

22. Trevor, J., Hilbert, D. and Schilit, B. Issues in personalizing shared ubiquitous devices. In Proc. of UbiComp 2002. Springer, 2002. pp. 56-72. 